\title{
Reunion of random walkers with a long range interaction: applications to polymers and quantum mechanics
}

\author{
Sutapa Mukherj婵 \\ Department of Physics and Meteorology, Indian Institute of Technology, Kharagpur 721 302, India \\ Somendra M. Bhattacharjee** \\ Institute of Physics, Bhubaneswar 751 005, India \\ Dipartimento di Fisica, Università di Padova, Via Marzolo 8, 35131 Padova, Italy
}

(October 25, 2018)

\begin{abstract}
We use renormalization group to calculate the reunion and survival exponents of a set of random walkers interacting with a long range $1 / r^{2}$ and a short range interaction. These exponents are used to study the binding-unbinding transition of polymers and the behavior of several quantum problems.

05.20.-y,36.20.-r, 03.65.-w,64.60.Ak
\end{abstract}

\section{INTRODUCTION}

The problem of reunion of interacting random walkers appears in many situations, directly or in disguise, such as e.g., in situations involving one-dimensional stringlike objects such as interfaces, steps on surfaces, in onedimensional quantum problems, in several types of polymer problems, wetting, etc. The issue quite often is the large-length scaling of the partition function of reunion (or survival) of a set of walkers all starting, say, at origin. For a purely short range repulsive interaction, this is the problem of "vicious walkers." Many of the vicious-walker reunion and survival exponents are known from exact calculations in one dimension [1].2], the renormalizationgroup (RG) approach [3, 1 t for general dimensions, and lattice models in two dimensions [5]. Recent studies of phase transitions on vicinal or miscut surfaces [6,7] required the scaling behavior of such partition functions in the presence of a long range interaction (namely $1 / r^{2}$ ) in addition to short range interactions. The effective interaction of steps on a vicinal surface has a long $r^{-2}$ tail [8] and recent studies on $\mathrm{Si}(113)$ surfaces indicate the presence of an attractive short range interaction [9] as well. Similar long range interactions occur in the onedimensional Calogero-Sutherland model [10] of interacting quantum particles, and as the angular momentum term in higher-dimensional quantum problems. The reunion and survival behaviors turn out to be the unifying feature in these wide varieties of problems. These motivated us to study the scaling limit of various partition functions in the presence of long range interactions, especially $r^{-2}$. We use these results for studying phase transitions of polymers and then show how many results of quantum problems can be recovered from such an analysis.

The success of the RG approach for short range interactions [3 5, 11] and the possibility of exact renormalization in the presence of long range interactions [12,7] allowed us to use the renormalization-group approach for this problem. It is known that the probability distribution function for a random walker can be represented by the Wiener integral, which as a path integral could, a la Edwards, represent a polymer. Any interaction of two or more walkers at the same time or step length would translate into an interaction among the polymers at the same contour length. For $d$-dimensional walkers, we would then get $(d+1)$-dimensional interacting directed polymers. We shall be using this polymer language throughout this paper.

Phase transitions in polymerlike systems by interactions at the same contour length occur, e.g., in the case of steps already mentioned, in flux lines in superconductors [13, in melting and unzipping of DNA 14 19, to cite a few. A directed polymer formulation then becomes quite natural. In this paper, our focus is mainly on the effect of the long range interaction on these phase transitions, recovering the results for the short range case as a special case. Furthermore, we exploit the relation between polymers and quantum problems to show the importance of reunion behaviors in wide varieties of problems in various dimensions [20].

In this paper, we recover several results known from exact solutions, illustrating how the reunion exponent armed with the renormalization group gives a unified approach to these problems. The RG approach can be extended to other types of interactions, especially other marginal interactions, for which exact solutions are not known, such as that required in the vicinal surface problem of Ref. [7]. There lies the merit of the approach of this paper.

The Hamiltonian and the general exponents of interest are introduced in Sec. II. The general RG approach is discussed in Sec. III, where the exponents are also calculated, with some of the details in the Appendix. These results are then used for polymer problems in Sec. IV and to quantum problems in Sec. V. The conclusion is given in Sec. VI. 


\section{HAMILTONIAN AND THE EXPONENTS}

The Hamiltonian for $p$-directed polymers with pairwise interaction [6,7] is

$$
H_{p}=\sum_{i}^{p} \int_{0}^{N} d z \frac{1}{2}\left(\frac{\partial \mathbf{r}_{i}(z)}{\partial z}\right)^{2}+\sum_{i>j} \int_{0}^{N} d z V\left(\mathbf{r}_{i j}(z)\right),
$$

where $\mathbf{r}$ is a $d$ dimensional position vector and $\mathbf{r}_{i j}(z)=$ $\mathbf{r}_{i}(z)-\mathbf{r}_{j}(z)$. The interaction potential is taken as

$$
V(\mathbf{r})=v_{0} \delta_{\Lambda}(\mathbf{r})+\frac{g}{|\mathbf{r}|^{\sigma}},
$$

and $\delta_{\Lambda}(\mathbf{r})$ is a delta function with a cutoff such that in Fourier space

$$
\begin{array}{rlrl}
\delta_{\Lambda}(\mathbf{q})=1 & & \text { for } \quad q \leq \Lambda \\
& =0 & & \text { otherwise }
\end{array}
$$

We shall use this cutoff for the whole potential. Our interest is in the asymptotic behavior of the following partition functions.

(a) Reunion of all the chains of length $N$ at a point $\mathbf{r}$,

$$
\begin{aligned}
& Z_{\mathrm{R}, p}(N, \mathbf{r}) \\
& \quad=\int \mathcal{D} R e^{-H_{p}} \prod_{i=1}^{p}\left[\delta^{d}\left(\mathbf{r}_{i}(0)\right) \delta^{d}\left(\mathbf{r}_{i}(N)-\mathbf{r}\right)\right] \\
& \quad \sim \quad N^{-\psi_{R, p}} ;
\end{aligned}
$$

(b) reunion anywhere,

$$
\begin{aligned}
\mathcal{Z}_{\mathrm{R}, p}(N) & =\int d^{d} r Z_{R, p}(N, \mathbf{r}) \\
& \sim N^{-\Psi_{R, p}}
\end{aligned}
$$

and (c) survival,

$$
\begin{aligned}
Z_{\mathrm{S}, p}(N) & =\int \mathcal{D} R e^{-H_{p}} \prod_{i=1}^{p}\left[\delta^{d}\left(\mathbf{r}_{i}(0)\right)\right] \\
& \sim N^{-\psi_{S, p}}
\end{aligned}
$$

where $\int \mathcal{D} R$ stands for the summation over all possible configurations of the polymers (path integrals). The constraint that all chains are tied together at the origin is represented by the product of the $\delta$ functions. In case (c), the ends at $z=N$ are free for all the chains.

In the above equations, the asymptotic behaviors define the basic exponents $\left(\psi_{R, p}, \Psi_{R, p}, \psi_{S, p}\right)$ of interest in this paper. These exponents are expected to be universal, independent of the detailed microscopic form of the polymers. Hence the choice of a continuum model.

The notation introduced above is used in Sec. III, where these exponents are calculated. However, in the subsequent sections on polymers and quantum problems, we need mainly $\Psi_{R, 2}$. We therefore adopt the notation

$$
\Psi \equiv \Psi_{R, 2} .
$$

Our primary interest is in the marginal case $\sigma=2$, though other values are also discussed briefly. The importance of $\sigma=2$ can be gauged if the Hamiltonian is considered as a quantum-mechanical system in imaginary time $t=i z$. The kinetic energy and the $r^{-2}$ interaction (like the angular momentum) scale in the same way for all dimensions. The $r^{-2}$ potential is the centrifugal barrier in quantum mechanics, while it occurs in surface problems as an effective interaction between steps induced by the elastic strains on the surface [8].

\section{A. Known exponents for the short range case}

For the noninteracting problem, $v_{0}=g=0$, the exponents follow from the Gaussian behavior of the chains. The partition function for a Gaussian chain of length $N$ is given by

$$
Z(\mathbf{R} N \mid \mathbf{0 0})=(2 \pi N)^{-d / 2} \exp \left(-R^{2} / 2 N\right),
$$

from which one obtains

$$
\psi_{\mathrm{S}, p}=0, \quad \psi_{\mathrm{R}, p}=\frac{p d}{2}, \quad \text { and } \quad \Psi_{\mathrm{R}, p}=\frac{(p-1) d}{2} .
$$

It is also known that for purely repulsive walkers (vicious walkers) in $d=1$ (i.e., with $g=0$ ) [1.2],

$$
\psi_{\mathrm{S}, p}=\frac{p(p-1)}{4}, \psi_{\mathrm{R}, p}=\frac{p^{2}}{2}, \text { and } \quad \Psi_{\mathrm{R}, p}=\frac{p^{2}-1}{2} .
$$

We earlier generalized the above results to $d=2-\epsilon$ for the short range case (i.e. $g=0$ ), by using $\mathrm{RG}$ and obtained [3,

$\psi_{\mathrm{S}, p}=\eta_{p}, \psi_{\mathrm{R}, p}=\frac{p d}{2}+2 \eta_{p}$, and $\Psi_{\mathrm{R}, p}=\frac{(p-1) d}{2}+2 \eta_{p}$,

where, with $\epsilon=2-d$,

$$
2 \eta_{p}=\left(\begin{array}{l}
p \\
2
\end{array}\right) \epsilon+3\left(\begin{array}{l}
p \\
3
\end{array}\right) \ln (3 / 4) \epsilon^{2}+\ldots
$$

For $d=2$, our RG yields $\mathcal{Z}_{\mathrm{R}, p} \sim N^{-(p-1)}(\ln N)^{-p(p-1)}$, which has since been obtained by the exact lattice calculations of Essam and Guttmann [5] for $p=2$. This lends further support to the validity of the renormalizationgroup approach.

That only one anomalous exponent is needed was shown explicitly in Refs. [3, [4]. This could be understood from the geometry that a reunion partition function can 
be thought of as two survival partition functions connected together.

In this paper, we calculate the anomalous part $\eta_{p}$ for the case with long range interaction at the one loop level. Since this one-loop RG generates exact results [12], we obtain exact exponents with nonzero $g$.

\section{RENORMALIZATION GROUP}

\section{A. Survival partition function}

To evaluate the survival exponent, we use a momentum-shell RG approach as used in Ref. 12. The renormalization process requires integrating out smallscale fluctuations, so that the effect of interactions of the two chains within a small distance is taken into account by redefining the parameters of the Hamiltonian. In this problem, in addition to the parameters of the Hamiltonian, we need to consider the survival partition function also.

In Fourier space, the renormalization of the interaction can be carried out using the effective interaction at the one-loop level

$$
V_{\text {eff }}(k)=V(k)-\int^{\Lambda} \frac{d^{d} q}{(2 \pi)^{d}} \frac{V(|\mathbf{k}-\mathbf{q}|) V(q)}{q^{2}} .
$$

The Fourier transform defined generically as

$$
F(\mathbf{k})=\int d^{d} r e^{i \mathbf{k} \cdot \mathbf{r}} G(\mathbf{r})
$$

requires an appropriate analytic continuation of the integral for singular potentials. In particular, the Fourier transform of $r^{-\sigma}$ has a singular part

$$
A \frac{k^{-d+\sigma}}{d-\sigma} \text { in general, }
$$

where

$$
A=2^{d-\sigma+1} \pi^{d / 2} \frac{\Gamma((d-\sigma+2) / 2)}{\Gamma(\sigma / 2)},
$$

and, for $\sigma=2$, this singular part takes a simpler form,

$$
\frac{1}{K_{d}} \frac{k^{2-d}}{d-2}
$$

where $K_{d}=S_{d} /(2 \pi)^{d}, S_{d}=2 \pi^{d / 2} / \Gamma(d / 2)$ being the surface of a $d$-dimensional unit sphere. A natural choice for the potential is therefore

$$
V(\mathbf{k})=v_{0}+\frac{A}{d-\sigma} g k^{\sigma-d} .
$$

Let us also introduce at this point the dimensionless parameters

$$
\tilde{u}=v L^{\epsilon}, \tilde{g}=K_{d} A g L^{2-\sigma}, \text { and } u=K_{d} \tilde{u}+\frac{\tilde{g}}{d-\sigma}
$$

with $v=v_{0}$ as the bare value of the short range coupling constant. Note that for $\sigma=2, g=\tilde{g}$.

By implementing a thin-shell integration over $\Lambda-d \Lambda<$ $k<\Lambda$ and then rescaling the cutoff back to $\Lambda(=1)$, the coupling constants' renormalization can be written in the form of recursion relations as

$$
\begin{aligned}
& L \frac{d u}{d L}=\epsilon u-u^{2}+\tilde{g}, \\
& L \frac{d \tilde{g}}{d L}=(2-\sigma) \tilde{g} .
\end{aligned}
$$

The thin-shell integration is analytic and therefore does not renormalize a singular $g$-type long range potential in the Hamiltonian. The flow equation of Eq. (3.6b) for $g$ then follows from dimensional analysis. The exactness of Eq. (3.6a) for $g=0$ and for $g \neq 0$ is discussed in Refs. [11,12. This marginality of $g$ is a reflection of the importance of an $r^{-2}$ potential in the Hamiltonian, as is well known in quantum mechanics. In the surface context, this marginality implies that the strength of interaction between two steps is invariant under scale transformation. Henceforth we consider $\sigma=2$, and use $g$ instead of $\tilde{g}$.

The flow equation for $u$ gives rise to two fixed points

$$
u_{s, u}^{*}=\frac{1}{2}\left[\epsilon \pm \sqrt{\epsilon^{2}+4 g}\right],
$$

where the subscripts $s$ and $u$ represent stable and unstable fixed points, respectively.

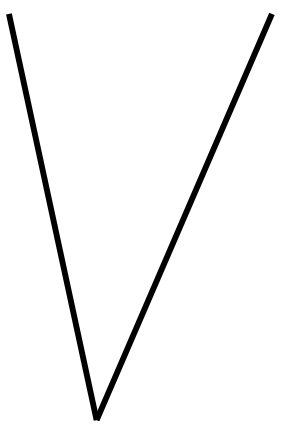

(a)

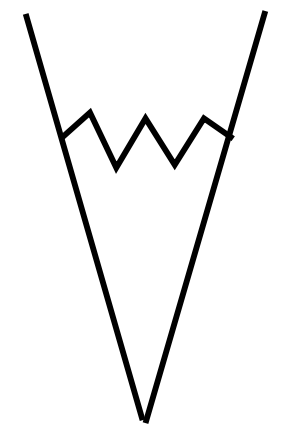

(b) mukherji-bhattacharjee

FIG. 1. Zeroth- and first-order diagrams for survival. The solid lines indicate the polymers and the wavy line interaction. 
For the survival partition function, the one-loop contribution is shown in Fig. 11. The loop contribution is similar to the one-loop case for the interaction, though here it is $O(u)$. The flow equation for $Z_{\mathrm{S}}$ is (see Appendix A for details)

$$
L \frac{d Z_{\mathrm{S}}}{d L}=-u Z_{\mathrm{S}}
$$

so that at the stable fixed point of Eq. (3.6a)

$$
\eta_{2}=u_{s}^{*} / 2
$$

This result can be generalized to any number of chains for which there will be a combinatorial factor $\left(\begin{array}{l}p \\ 2\end{array}\right)$ from the pairwise interaction to yield

$$
\eta_{p}=\frac{1}{2}\left(\begin{array}{l}
p \\
2
\end{array}\right) u_{s}^{*} .
$$

This exact relation for $p=2$ is a consequence of the connection between the coupling constant $u$ (vertex function) and the survival partition function. The interaction $u$ can be thought of as two $p=2$ survival partition functions joined at the node so that renormalization of $u$ is a product of the renormalizations of two survival partition functions.

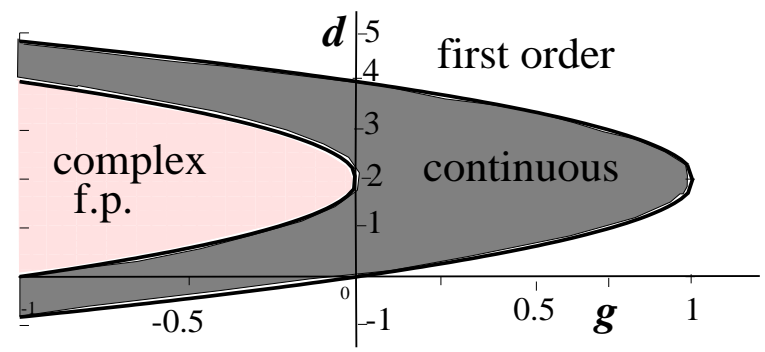

FIG. 2. The locus of points in the $d$ vs $g$ plane for reunion exponent $\Psi_{R, 2}=1$ and $\Psi_{R, 2}=2$. The shaded region is the region for continuous transition. The transition is first-order outside it. The lightly shaded region has complex-conjugate fixed points that are of importance in the context of non-hermitian Hamiltonians.

The reunion-anywhere exponent for $d=1$ is given by

$$
\Psi_{\mathrm{R}, p}=\frac{p-1}{2}+\left(\begin{array}{l}
p \\
2
\end{array}\right) u_{s}^{*} .
$$

A special case of this is $\Psi_{R, 2}=\frac{1}{2}+u_{s}^{*}$. For only short range interactions (i.e., $g=0$ ), $u_{s}^{*}=1$ and one gets back the vicious walker exponent of Huse and Fisher $\left[\Psi_{R, p}=\left(p^{2}-1\right) / 2\right]$ [1]:2]. One notes that the anomalous part is just the combinatorial factor. The general results for all three reunion and survival exponents are obtained by substituting Eq. (3.10) for $\eta_{p}$ in Eq. (2.7a). We quote the exact result for $d=2-\epsilon$ and $p=2$,
$\Psi \equiv \Psi_{R, 2}=\frac{d}{2}+\frac{\epsilon+\sqrt{\epsilon^{2}+4 g}}{2}=1+\frac{\sqrt{\epsilon^{2}+4 g}}{2}$.

This is used in the following sections.

A few things are to be noted here. First, the fixed points become complex for $g<-\epsilon^{2} / 4$, in particular for $g<-1 / 4$ for $d=1$. For this borderline case $d=2 \pm 2 \sqrt{-g}$, the reunion exponent is $\Psi_{R, 2}=1$. The locus of the points in the $d$ vs $g$ plane for $\Psi_{R, 2}=1$ and $\Psi_{R, 2}=2$ are shown in Fig. 2. As we show in Sec. IV, these lines are very special.

\section{BINDING-UNBINDING TRANSITION OF POLYMERS}

Let us consider two directed polymers, the $p=2$ case of Eq. 2.1a). The fixed-point diagram of Fig. IVA shows that at $d=1$, for a given $g>-1 / 4$, there will be a binding-unbinding transition as $v$ is changed (see Fig. 1 of Ref. [7]). The transition is defined by the unstable fixed point while the stable fixed point describes the "high-temperature" phase. Several features of this transition have been discussed in Ref. [12] in the renormalization group framework and in Ref. 21]. We show here that certain unresolved issues (e.g., order of transition) in the RG framework can be sorted out by using the reunion exponent, thereby gaining a complete picture of the problem. The problem can be anticipated by noting that the exponent $\nu$ associated with the diverging length scale, as the transition is reached, approaches the limit $\nu=1 / 2$ as $g \rightarrow 3 / 4$. Care should, therefore, be exercised in drawing conclusions from the flow diagram. We discuss now how the behavior at the stable fixed points determines the phase transition at the unstable fixed point.

For generality, we develop the procedure in a general way and then derive the results for the $1 / r^{2}$ interaction, especially for $p=2$ (two-chain case). The internal consistency is also shown by a finite-size scaling argument. That the exact results are recovered corroborates the utility of the RG approach, coupled with the reunion behavior, for other interactions as well.

\section{A. General approach}

In order to study the nature of the transition, we adopt the procedure of Ref. [1] . The partition function is obtained by summing over all configurations of the two chains. Our main interest is in the phase-transition behavior and therefore only long-distance behaviors will be considered. The configurations can be characterized by the alternate sequence of domains of bound (A) and unbound (bubble, B) regions as shown Fig. IV A. The bound regions correspond to the left side of the unstable fixed point of the flow diagram (the coupling constant flows to $-\infty$ ), while the bubbles correspond to the 
high-temperature phase characterized by the stable fixed point.

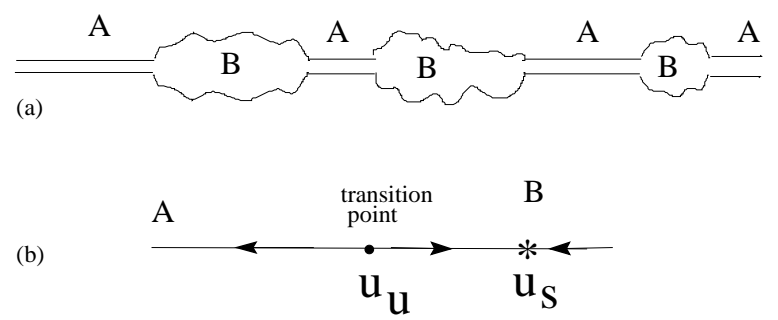

Mukherii-bhattacharjee, Fig 3

FIG. 3. (a) A general configuration of the two polymers, consisting of bound (A) and unbound (B) regions. (b) A generic fixed-point diagram for $u$ from the RG flow. See also Fig. 1 of Ref. [7].

Let us denote the partition function for a segment of length $N$ of type $\mathrm{X}$ by $Q_{N}^{\mathrm{X}}$. We assign a weight $v$ to each $\mathrm{A}-\mathrm{B}$ and $\mathrm{B}-\mathrm{A}$ junction, and for simplicity we consider cases in which the chains start and end in A-type domains. This extra factor $v$ is to take care of the deviation from the strict A- or B-type behavior at the junctions. The total partition function can be written as

$Q_{N}^{\mathrm{T}}=Q_{N}^{\mathrm{A}}+\int_{0}^{N} d z_{2} \int_{0}^{z_{2}} d z_{1} Q_{N-z_{2}}^{\mathrm{A}} v Q_{z_{2}-z_{1}}^{\mathrm{B}} v Q_{z_{1}}^{\mathrm{A}}+\ldots$

To exploit the convolution nature of the terms of the series, we go over to the grand-canonical ensemble with respect to the chainlength. This is equivalent to taking the Laplace transform of the partition functions with respect to $N$. Defining the Laplace transform as

$$
G^{\mathrm{X}}(s)=\int_{0}^{\infty} e^{-s N} Q_{N}^{\mathrm{X}} d N
$$

where $\mathrm{X}$ is $\mathrm{A}, \mathrm{B}$ or $\mathrm{T}$, the above series can be written in the form of a geometric series and can be summed to obtain

$$
G^{\mathrm{T}}(s)=\frac{G^{\mathrm{A}}(s)}{1-v^{2} G^{\mathrm{A}}(s) G^{\mathrm{B}}(s)}
$$

The existence of the thermodynamic limit ensures that $G^{\mathrm{X}}(s)$ is analytic for $s>f_{X}$, where $f_{X}=$ $\lim _{N \rightarrow \infty} N^{-1} \ln Q_{N}^{\mathrm{X}}$, and $G^{\mathrm{X}}(s) \rightarrow 0$ as $s \rightarrow \infty$. In other words, the largest real singularity of $G^{\mathrm{X}}(s)$ in the complex- $s$ plane determines the corresponding limiting free energy per unit length [22]. For $G^{\mathrm{T}}(s)$, this could either be the singularity of $G^{A}$ or $G^{B}$ or may come from the vanishing of the denominator of Eq. (4.3), i.e. the solution of

$$
v^{2} G^{\mathrm{B}}(s)=1 / G^{\mathrm{A}}(s) .
$$

In case $G^{A}$ or $G^{B}$ diverges, then the denominator of Eq. (4.3) vanishes for a larger value of $s$, and then it is the root of Eq. (4.4) that is the relevant one. In such a situation, the free energy will not have any singularity. It follows that it is only the nondiverging branch points that could lead to phase transitions.

For the A-type region, the partition function $Q_{N}^{\mathrm{A}}=$ $e^{-\beta \varepsilon N}$, where $\beta$ is the inverse temperature. Its Laplace transform has a simple pole. Therefore, the singularity of $G^{A}$ is not important for our discussion. Consequently, it is the partition function of the bubbles (high-temperature phase) that determines the behavior of the chains 2325. The partition function of the bubble at the stable fixed point for large $N$ is given by

$$
Q_{N}^{B} \approx \frac{q e^{-N \sigma_{0}(T)}}{N^{\Psi}}
$$

where the reunion-anywhere exponent $\Psi$ at the stable fixed point appears. Here and in the following discussion, for convenience, we have removed the subscripts of the reunion exponent introduced in Sec. II [see Eq. (2.3)]. The Laplace transform of this partition function has a singularity at $s=\sigma_{0}(T)$, and the nature of the singularity depends on the value of the reunion exponent, $\Psi$. There is a divergence at this singularity if $\Psi<1$. A phase transition therefore occurs only for $\Psi \geq 1$. The details of the graphical solution, for the discrete case, can be found in Ref. [1].

For $\Psi>1$, the high temperature phase is described by the root of Eq. (4.4) and the transition is given by the temperature $T=T_{c}$ at which the root of Eq. (4.4) coincides with the singularity of $G^{B}(s)$. Defining the deviation of temperature as $t=T_{c}-T$, the free energy [from the root of Eq. 4.4] can be written as

$$
\begin{aligned}
f_{\mathrm{T}} \approx \sigma_{0}(T)-|t|^{1 /(\Psi-1)} \text { for } 1<\Psi<2, \\
\approx \sigma_{0}(T)-\sum_{1}^{m-1} a_{j} t^{j}+|t|^{(\Psi-m)} \\
\quad \text { for } m<\Psi<m+1 .
\end{aligned}
$$

We find a critical behavior for $1<\Psi<2$ but a firstorder transition for $\Psi>2$. In case of a critical behavior, the specific-heat exponent can be read off from the free energy as

$$
\alpha=2-(\Psi-1)^{-1}=\frac{2 \Psi-3}{\Psi-1} .
$$

\section{B. $d=1$}

The crucial feature in the approach developed in the preceding subsection is the alternate sequence of the two types of "bubbles" and therefore the results can be used, 
for example, in the case of unbinding of $p$ chains with $p$-body interaction [11, 4 and other cases. To take advantage of previous studies, we concentrate on the two-chain problem.

Let us now consider the case of two directed polymers in $(1+1)$ dimensions, for which we obtained $\Psi=$ $(1+\sqrt{1+4 g}) / 2$. The fixed points (see Fig. IV A) indicate that there is a binding-unbinding transition at the unstable fixed point for any given $g$ as $u$ is varied. For any $u_{0}<u_{\mathrm{u}}^{*}$, the renormalized flow goes to $-\infty$, so that a length scale can be identified from $u\left(l^{*}\right) \rightarrow-\infty$. From this, as $u_{0} \rightarrow u_{\mathrm{u}}^{*}$, the diverging length scale comes out as $\left(t \equiv \Delta u=u-u_{u}^{*}\right)$

$$
\xi_{\perp} \sim|t|^{-\nu_{\perp}} \quad \text { with } \quad \nu_{\perp}=\frac{1}{u_{s}^{*}-u_{u}^{*}} .
$$

For $d=1$, we have

$$
\nu_{\perp}=\frac{1}{\sqrt{1+4 g}} .
$$

This shows that $\nu_{\perp}=1 / 2$ at $g=3 / 4$, a signature of something special happening there. The reunion exponent, coupled with the analysis of the preceding subsection, tells us that there cannot be a phase transition for $g<-1 / 4$, a fact corroborated by the fixed-point diagram (see Fig. 1 of Ref. [7]). A critical behavior is expected for $3 / 4 \geq g \geq-1 / 4$ and a first-order transition for $g>3 / 4$.

For $-1 / 4 \leq g \leq 3 / 4$, which includes the pure short range $g=0$ case, the average fraction of the length in the bound state is given by

$$
\Theta(t) \sim \frac{\partial f}{\partial t} \sim|t|^{\beta}
$$

This defines the order-parameter exponent $\beta$. This exponent and the specific-heat exponent are given by

$$
\beta=\frac{2-\Psi}{\Psi-1}=\frac{2-\sqrt{1+4 g}}{\sqrt{1+4 g}}, \text { and } \alpha=2 \frac{\sqrt{1+4 g}-1}{\sqrt{1+4 g}} .
$$

Note that $\alpha=1$ at $g=3 / 4$, a requirement for a firstorder transition.

The bubble lengths have fluctuations and this gives the measure of the diverging length scale parallel to the chain as

$$
\xi_{\|} \sim|t|^{-\nu_{\|}} \quad \text { with } \quad \nu_{\|}=2 \nu_{\perp}
$$

Note that the hyperscaling relation $d \nu_{\|}=2-\alpha$ is obeyed by these nonuniversal exponents with $d=1$. This is because the free-energy density is the free energy per unit length of the polymers and not the unit $(d+1)$ dimensional area.

If $g>3 / 4, \Theta(t) \rightarrow$ const. as $t \rightarrow 0-$. This indicates a first-order transition. The longitudinal length-scale exponent is $\nu_{\|}=1$, i.e., it sticks to its value at $g=3 / 4$.
However, the free energy as given by Eq. (4.6a) shows a weak singularity that will be reflected in the divergence of an appropriate higher derivative. One therefore finds a rather unusual first-order transition with weak singularities and diverging length scales [21]. Such scales can be determined from the higher cumulants of the length fluctuations of the bubbles. We discuss these issues in the next section in the context of the equivalent quantum problem.

$\Psi$ increases with $g$ and every-time $\Psi$ crosses an integer, the diverging derivative shifts by 1 . These special values are $g=(m-1)^{2}-1 / 4$.

At $g=-1 / 4$, two fixed points $u_{s}^{*}$ and $u_{u}^{*}$ merge at a common value $u^{*}=1 / 2$. The bound phase corresponds to the $u_{0}<1 / 2$ region and the transverse length scale diverges as

$$
\xi_{\perp} \sim \exp \left[1 /\left(1 / 2-u_{0}\right)\right]
$$

However a Kosterlitz-Thouless-type behavior is observed if $g$ is varied 12 .

1. Finite size scaling for the critical point

We have derived the exponents for the critical point from our results of the stable fixed point or the hightemperature phase. An independent verification of the exponents comes from a finite-size scaling argument used strictly in the critical region characterized by the unstable fixed point. The reunion describes the contacts of the two chains and therefore the number of contacts at the unstable fixed point would have a finite-size scaling behavior ( $L$ is a length along the chain)

$$
\Theta_{L} \sim L^{-\Psi^{\{u\}}} \sim t^{\Psi^{\{u\}} / \nu_{\|}}
$$

which identifies

$$
\beta=\frac{2-\Psi^{\{s\}}}{\Psi^{\{s\}}-1}=\frac{\Psi^{\{u\}}}{\nu_{\|}}
$$

where we have introduced the superscripts $\{s\}$ and $\{u\}$ to distinguish the values of the exponent at the stable and unstable fixed points, respectively. [ $\Psi$ of Eq. (4.11) is $\Psi^{\{s\}}$ here.] Using the unstable fixed point value in Eq. (3.11), we do see this equality to be true because

$$
\Psi^{\{u\}}=1-\frac{\sqrt{1+4 g}}{2} .
$$




$$
\text { C. } d \neq 1
$$

For $1<d<2$, i.e., $d=2-\epsilon$ with positive $\epsilon$, the results of the preceding subsection can be repeated by using $\Psi$ of Eq. (3.12). The reunion exponent requires $g>-\epsilon / 4$ (so that $\Psi>1$ ) for a phase transition that is identical to the condition of the real fixed point of the flow equation. The length-scale exponent becomes equal to $1 / 2$ at $g=d(4-d) / 2=\left(4-\epsilon^{2}\right) / 2$, which coincides with the value of $g$ at which $\Psi=4$. The exponents satisfy the general scaling relations of Eqs. (4.12) and (4.15).

The stability of the fixed points flips at $d=2$. The results for $d>2$ can be obtained by doing an analytic continuation from $\epsilon>0$ to $\epsilon<0$. For example, on the stable branch now $\Psi=1+\sqrt{\epsilon^{2}+4 g} / 2$. Figure 2 shows the regions of critical and first-order transition in the $d$ vs- $g$ plane.

\section{QUANTUM PROBLEM}

The Hamiltonian of Eq. 2.1a represents two quantum particles either in the path-integral representation in imaginary time or as a statistical mechanical problem at $T=0$. In the center-of-mass frame, the equivalent Schrödinger equation for $\sigma=2$, where $v_{0}(r)$ is the short range part of the interaction, is

$$
-\nabla^{2} \psi(\mathbf{r})+\left[v_{0}(\mathbf{r})+\frac{g}{r^{2}}\right] \psi(\mathbf{r})=E \psi(\mathbf{r}) .
$$

For zero energy, the radial part of the wave function can be written as

$$
R(r)=\exp \left[\int \frac{u(r)}{r} d r\right]
$$

with $u(r)$ satisfying

$$
r \frac{d u}{d r}=(2-d) u-u^{2}+g
$$

Taking $l=\ln (r / a)$, we can recast the above equation in a form resembling the RG flow equation. In other words, the RG flow equation at long distances determines the radial part of the zero-energy wave function.

We consider the transition point itself, which corresponds to the unstable fixed point. In case $u$ of Eq. (5.3) reaches a fixed point, the wave function has an algebraic tail as $R(r) \sim|r|^{u^{*}}$.

The wave function $R(r)$ satisfying the Schrödinger equation is analogous to the partition function of a polymer starting from origin to the point $r$. Its behavior is similar to the survival partition function because in the process the particle may feel (in a perturbative approach) the potential any number of times. The scaling that time and space are related by $[N]=[r]^{2}$ then gives, via the scaling of the survival partition function,

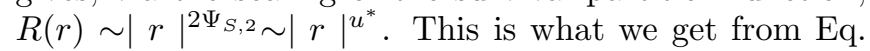
(5.3).
At $d=1$, we then get at $u=u_{\mathrm{u}}^{*}$ [the unstable fixed point of Eq. (3.7) with $\epsilon=1] R(r) \sim|r|^{u_{\mathrm{u}}^{*}}$. The moments of scalar $r^{2}=\mathbf{r} \cdot \mathbf{r}$, defined for general $d$ by

$$
<r^{2 p}>=\frac{\int(\mathbf{r} \cdot \mathbf{r})^{p} R(r)^{2} r^{d-1} d r}{\int R(r)^{2} r^{d-1} d r}
$$

would depend on the long tail of the wave function. The wave-function itself is not normalizable for $u_{\mathrm{u}}^{*}>-1 / 2$, i.e., for $g<3 / 4$. This is a signature of an unbound state. In such a situation, by tuning the short range part of the potential from below, one can get a bound state arbitrarily close to zero energy. As the zero-energy state is reached, the length scale that measures the boundedness or localization of the state increases without bound. Obviously, all the moments are divergent. This is the quantum picture of the criticality discussed in the preceding section for polymers.

For $g>3 / 4$, the zero-energy wave function is normalizable. This means a bound state with zero energy but with a long tail of the wave function. In this region, the bound state can also be made to approach the zero energy state but there will always be a finite length scale coming from the finite moments of the wave-function. This is a first-order transition, but with a difference. The moments of $r^{2}$ are finite for $p<(\sqrt{1+4 g}) / 2-1$, i.e., for $p<\Psi-2$, and any value of $p<\Psi-2$ may be used to define a finite length scale. However, the divergence of the higher moments indicates some remnants of the "criticality." The integer values of the reunion exponent, which show up in the free energy in Eq. (4.6a), also make their presence felt here as the special values at which a new integer moment becomes finite.

We immediately see that all the moments of $r^{2}$ are diverging for $u_{\mathrm{u}}^{*}>-1 / 2$, and the first $p$ moments are finite for

$$
g>(p+1)^{2}-1 / 4
$$

For $p$ violating this criterion, using a finite-size scaling analysis (i.e., by cutting off the integral for large $r$ by the length scale), one gets

$$
\nu_{p}=\frac{1}{4 p}(2 p+2-\sqrt{1+4 g}) .
$$

That there is a diverging length scale coming from higher moments gives an a posteriori justification of the RG analysis based on fixed points.

The states with $E<0$ always have at least exponential (or faster) decays of the wave function at large distances. This rapid decay ensures finiteness of all moments. However, at the transition point, the possibility of diverging moments arises because of the power-law ("critical") decay of the wave function. 


\section{A. Short range interaction, general $d$}

We now show that the one-dimensional exact results for the reunion behavior can be made to bear upon the higher-dimensional problems also. In the process, we recover many results obtained earlier from detailed exact solutions for each $d$ by using the properties of special functions 26,27.

Let us consider a short range central potential $V(\mathbf{r})$. Defining $\phi(r)=r^{(d-1) / 2} R(r)$, where $R(r)$ is the radial part of the wave function, the Schrödinger equation in $d$ dimensions can be written as [26]

$$
-\frac{d^{2} \phi}{d r^{2}}+\left(\frac{A_{l}(d)}{r^{2}}+V(r)\right) \phi(r)=E \phi,
$$

where $A_{l}(d)=(d+2 l-3)(d+2 l-1) / 4$ is the coefficient of the angular momentum ( or centrifugal) barrier, $l$ being the integer angular momentum quantum number. Note that the factor $r^{(d-1) / 2}$ makes the integral over $r$ the same as that of the one-dimensional problem. The $d$-dimensional problem is then reduced to the one dimensional problem and the exact results for $d=1$ for various $g$ as obtained in the previous subsection can be used to get the features as $d$ and $l$ are changed. The ground state is obtained from $l=0$ for $d \geq 1$. For other values of $d$, $l$ is to be chosen such that $1-2 l \leq d<3-2 l$. (The choice makes the minimum centrifugal barrier.) By using our results, we see that for $A_{l}(d)<3 / 4$, there is no zero-energy bound state, though the state can be reached continuously from below. Substituting the expression for $A_{l}(d)$, we find $\Psi=\frac{d}{2}+l$ for $d>2$. This shows that for $l=0$ and $1<d<4$, there can be no bound state at zero energy. As the short range parameter is tuned, the localization length of the bound state diverges with an exponent

$$
\nu_{\perp}=\frac{1}{2} \nu_{\|}=\frac{1}{|d-2|} \quad \text { for } 1 \leq d<4,
$$

with $\Psi=2$ at $d=4$. For $d<1$, the ground state comes from $l=1$, so that

$$
\nu_{\perp}=\frac{1}{|d|} \quad \text { for } \quad-1 \leq d<1 .
$$

Similarly, $\nu_{\perp}=1 /|d+2|$ for $-3 \leq d<-1$ when $l=2$. In general,

$$
\nu_{\perp}=\frac{1}{|d+2 l-2|} \quad \text { for } 1-2 l \leq d<3-2 l .
$$

For all $d<4, \nu_{\|} / \nu_{\perp}=2$. For $d>4$, we find from Eq. (5.5) that the $p$ th moment of $r$ will be finite [27] if $p<d-4$, and for $p>d-4$ one gets

$$
\nu_{p}=\frac{1}{2}-\frac{d-4}{2 p} .
$$

\section{CONCLUSION}

In conclusion, we have investigated the reunion of random walkers having both short range and long range interactions. By using a momentum-shell renormalizationgroup technique, the reunion and survival exponents have been calculated. The exponent $\Psi \equiv \Psi_{R, 2}$ for polymers has been evaluated at the unbound phase and at the binding-unbinding transition point represented by the stable and the unstable fixed points, respectively, in the coupling constant space. The value of this exponent in the unbound phase is crucial in determining the nature of the binding-unbinding transition [21, 12, 7. This transition is critical for $1<\Psi<2$ and first order with higher moments diverging for $\Psi>2$. Since $\Psi$ is explicitly dependent on the strength of the long range interaction $g$, the order of the phase transition depends on this parameter. See Fig. 2. For example, at $d=1$, the dimension more relevant in the context of the experimental observation of vicinal surfaces [7], one finds a first-order transition for $g>3 / 4$ and criticality for $-1 / 4<g<3 / 4$. In the quantum-mechanical picture, the different nature of the phase transition is reflected in the approach of the bound state to the zero-energy state as the short range part of the potential is tuned. For $g<3 / 4$, one can get the bound state arbitrarily close to the zero-energy state with a diverging length scale as the gap vanishes, whereas for the first-order case $(g>3 / 4)$, the length scale remains finite. In the latter case, it is possible to define diverging length scales from higher moments [see Eq. (5.6)]. This one-dimensional case can further be extended to the case of a quantum particle subjected to a short range potential in dimensions $d \neq 1$ with the centrifugal barrier playing the role of the long range potential. The fact that all the details and the nuances of the $r^{-2}$ interaction problem known from exact solutions [10,21,26,27] could be recovered in a unified manner via the reunion behavior lends credence to the general approach developed in this paper. Our method can be used for other problems and interactions as well.

\section{APPENDIX A: RENORMALIZATION OF THE SURVIVAL PARTITION FUNCTION}

In the noninteracting case, the survival partition function $Z_{s}$ for Gaussian walkers is unity. The anomalous exponent of $N$ in the survival partition function appears due to the interaction among the chains. The nontrivial contribution of this interaction is apparent from the one-loop $O(u)$ term, which is shown in Fig 1 The contribution of this diagram is

$$
\begin{array}{r}
\int_{0}^{N} \int d \mathbf{r}_{1} d \mathbf{r}_{2} Z\left(z, \mathbf{r}_{1} \mid 0, \mathbf{0}\right) Z\left(z, \mathbf{r}_{2} \mid 0, \mathbf{0}\right) \times \\
\left(v_{0} \delta\left(\mathbf{r}_{1}-\mathbf{r}_{2}\right)+\frac{g}{\left|r_{1}-r_{2}\right|^{\sigma}}\right)
\end{array}
$$


In the Fourier space, this term appears as

$$
\int_{0}^{N} \int d \mathbf{p} Z(z, \mathbf{p}) Z(z,-\mathbf{p})\left[v_{0}+v_{\mathrm{lr}}(p)\right]
$$

where $v_{\operatorname{lr}}(p)=\frac{A g p^{\sigma-d}}{d-\sigma}$. By using $Z(z, \mathbf{p})=\exp \left[-p^{2} z / 2\right]$, we perform integration only over an outer shell of radii $\Lambda$ and $\Lambda(1-\delta)$, where $\delta$ is a very small parameter. This is done essentially to integrate out the small-scale fluctuations. In the large- $N$ limit, the contribution of the above term after the momentum-shell integration is

$$
\Lambda^{-\epsilon} \delta K_{d}\left[v_{0}+v_{\operatorname{lr}}(\Lambda)\right]
$$

By taking this term into account, one can express $Z_{s}$ viewed at a larger length scale $(\Lambda-\delta \Lambda)^{-1}$ in terms of $Z_{s}$ viewed at a smaller resolution as

$$
Z_{s}(\Lambda-\delta \Lambda)=Z_{s}(\Lambda)-Z_{s}(\Lambda) K_{d} \Lambda^{-\epsilon}\left[v_{0}+v_{\operatorname{lr}}(\Lambda)\right],
$$

where only the cutoff dependence is shown explicitly. This equation can further be transformed into a differential equation form

$$
\frac{d \ln Z_{s}}{d \Lambda}=-u,
$$

where $L=\Lambda^{-1}$. By using the fixed-point value for $u$, one obtains the exponent given in Eq. (3.9).

* email: sutapa@phy.iitkgp.ernet.in.

** email: somen@iopb.res.in.

[1] M. E. Fisher, J. Stat. Phys. 34, 667 (1984).

[2] D. A. Huse and M. E. Fisher, Phys. Rev. B29, 239 (1984).

[3] S. Mukherji and S. M. Bhattacharjee, J. Phys A 26, L1139 (1993)

[4] S. Mukherji and S. M. Bhattacharjee, Phys. Rev. E 48, 3427 (1993); 52, 3301(E) (1995).

[5] J. W. Essam and A. J. Guttmann, Phys. Rev. E 525849 (1995).

[6] S. M. Bhattacharjee, Phys. Rev. Lett. 76, 4568 (1996). Note that $g$ and $h$ of this reference should be the same. See also M. Lassig, ibid 77, 526 (1996), and V. B. Shenoy, S. Zhang, and W. F. Saam, ibid 81, 3475 (1998); condmat/9901153. The last reference makes a comment, after Eq. (53), on the nonapplicability of the continuum theory for $g>3 / 4$. This is not correct. The proper use of RG in this region has already been shown in Ref. [7] below. The present paper provides more details.

[7] S. M. Bhattacharjee and S. Mukherji, Phys. Rev. Lett, 83, 2374 (1999).
[8] V. J. Marchenko and A. Ya Parshin, Sov. Phys. JETP 52, 129 (1981); W, Kohn and K. H. Lau, Sol. State Commun. 18, 553 (1976).

[9] (a) S. Song and S. G. J. Mochrie, Phys. Rev. Lett. 73, 995 (1994); S. Song and S. G. J. Mochrie, Phys. Rev. B 51, 10068 (1995).

(b) M. Yoon et al. Surf. Sci. 411, 70 (1998).

[10] F. Calogero, J. Math. Phys. 10, 2191 (1969); 10, 2197 (1969); B. Sutherland, ibid 12, 246 (1971); 12, 251 (1971); Phys. Rev A 4, 2019 (1971); 5, 1372 (1972). For conections with random matrix theories, see, e.g., P. Shukla, Phys. Rev. E. 62, 2098, (2000).

[11] S. M. Bhattacharjee and J. J. Rajasekaran, Phys. Rev. A 46, R703 (1992); S. M. Bhattacharjee, Physica A 186, 183 (1992).

[12] E. B. Kolomeisky and J. P. Straley, Phys. Rev. B 46, 12664 (1992). E. B. Kolomeisky Phys. Rev. Lett. 73, 1648 (1994).

[13] See, e.g., S. Mukherji and T. Nattermann, Phys. Rev. Lett. 79, 139 (1997), and references therein.

[14] S. M. Bhattacharjee, cond-mat/9912297. J. Phys. A33,L423 (2000); 33, 9003(E) (2000). D. Lubensky and D. R. Nelson, Phys. Rev. Lett. 85, 1572 (2000).

[15] S. M. Bhattacharjee, cond-mat/0010132

[16] A. Maritan, E. Orlandini and F. Seno, Preprint (2000).

[17] D. Marenduzzo, A. Trovato and A. Maritan, condmat/0101207

[18] M. S. Causo, B. Coluzzi and P. Grassberger, Phys. Rev. E 62, 3958 (2000).

[19] S. M. Bhattacharjee and S. Mukherji, Phys. Rev. Lett. 70, 49 (1993); S. Mukherji and S. M. Bhattacharjee ibid 70, 3359 (E) (1993). Phys. Rev.E 48, 3483 (1993).

[20] The connection between reunion and overlaps of directed polymers in a random medium has been noted earlier. See, S. Mukherji, Phys. Rev. E50, 2407 (1994), and S. Mukherji and S. M. Bhattacharjee, Phys. Rev. B 53, 6002 (1996).

[21] R. Lipowsky, Europhys. Lett. 15, 703 (1991). For a review, see, e.g., G. Forgacs, R. Lipowsky, and Th. Nieuwenhuizen, in Phase Transitions and Critical Phenomena, edited by C. Domb and J.L. Lebowitz (Academic, New York, 1991), Vol. 14.

[22] This is analogous to the condition determining the radius of convergence of the generating function for the discrete case of Ref. [1]. Note that this corresponds to the root closest to origin in the formulation of Ref. [犋.

[23] S. Lifson, J. Chem. Phys. 40, 3075 (1964).

[24] D. Poland and H. A. Scheraga, J. Chem. Phys. 45, 1464 (1966).

[25] M. E. Fisher, J. Chem. Phys. 45, 1469 (1966)

[26] R. Balian and G. Toulouse, Ann. Phys.(N. Y.) 83, 28 (1974).

[27] R. Lipowsky and Th. M. Nieuwenhuizen, J. Phys. A 21, L89 (1988); R. K. P. Zia, R. Lipowsky, and D. M. Kroll, Am. J. Phys. 56, 160 (1988). 